\title{
The Lotus intrinsic ethylene receptor regulates both symbiotic and non-symbiotic responses
}

\author{
Kana Miyata', Tomomi Nakagawa ${ }^{2,3, *}$ \\ ${ }^{1}$ Department of Life Sciences, School of Agriculture, Meiji University, Kawasaki, Kanagawa 214-8571, Japan; ${ }^{2}$ Division of \\ Symbiotic Systems, National Institute for Basic Biology, Okazaki, Aichi 444-8585, Japan; ${ }^{3}$ Division of Biological Science, \\ Graduate School of Science, Nagoya University, Nagoya, Aichi 464-8602, Japan \\ *E-mail:nkgwtmm@nibb.ac.jp Tel \& Fax: +81-564-55-7563
}

Received November 4, 2015; accepted February 15, 2016 (Edited by T. Mizoguchi)

\begin{abstract}
The phytohormone ethylene regulates plant growth, development, and responses to both biotic and abiotic stresses. Ethylene also negatively regulates rhizobial symbiosis in legumes, although the intrinsic ethylene signaling components in legumes are still largely unclear. We report a novel ethylene insensitive mutant named Ljetr1 from the model legume Lotus japonicus. Ljetr1 showed growth tolerance to high concentrations of 1-amino-cyclopropane-carboxylic acid, the biosynthetic precursor of ethylene. Petal senescence and abscission were delayed and number of nodules was slightly increased compared to wild-type. Mapping analysis and genome sequencing showed that Ljetr1 bears a mutation in the ethylene-binding domain of the Arabidopsis ETR1 homolog. Our results suggest that the Lotus intrinsic ethylene receptor LjETR1 regulates the ethylene signaling pathway in both non-symbiotic and legume-specific symbiotic responses.
\end{abstract}

Key words: Ethylene, ETR1, Lotus japonicus, nodulation, symbiosis.

Leguminous plants establish a mutualistic symbiosis with rhizobial bacteria and form specialized root organs, known as nodules. In this symbiosis, rhizobia convert atmospheric nitrogen to ammonia and provide it as nutrient to host plants in exchange for photosynthates. This symbiosis enables host legume plants to grow vigorously under nitrogen-nutrient deficient conditions. On the other hand, excessive nodulation impairs host plant growth, because establishment and maintenance of the symbiosis has a high cost in photosynthates. Therefore, leguminous host plants strictly regulate the number of nodules and maintain an appropriate level of symbiosis (Suzaki et al. 2015).

Application of the gaseous phytohormone ethylene, or its biosynthetic precursor 1-amino-cyclopropanecarboxylic acid (ACC), strongly inhibits nodulation in a wide variety of leguminous plants including soybeans (Schmidt et al. 1999), Medicago truncatula (Penmetsa and Cook 1997), Macroptilium atropurpureum and Lotus japonicus (Nukui et al. 2000). Conversely, a treatment with an ethylene biosynthesis inhibitor, $\mathrm{L}-\alpha-(2-$ aminoethoxyvinyl)-glycine (AVG), slightly promotes the number of rhizobial infections and nodulations (Nukui et al. 2000, Oldroyd et al. 2001, Penmetsa and Cook 1997). These pharmacological studies suggest that the ethylene signaling pathway has a role in the negative regulation of nodulation.

The ethylene signaling pathway in plants has been extensively studied in the non-legume Arabidopsis. In Arabidopsis, ethylene is recognized at the endoplasmic reticulum (ER) membrane by a family of ethylene receptors, consisting of ETR1 (ETHYLENE RESPONSE1), ETR2, ERS1 (ETHYLENE RESPONSE SENSOR1), ERS2, and EIN4 (ETHYLENE INSENSITIVE4) that share similarities with bacterial two-component histidine kinases (reviewed in Guo and Ecker 2004). In the absence of ethylene, these ethylene receptors redundantly suppress ethylene responses. Recognition of ethylene inactivates the ethylene receptors and accordingly results in the cleavage of EIN2, an integral ER membrane protein consisting of an $\mathrm{N}$-terminal transmembrane domain and a C-terminal domain containing an unknown motif (Alonso et al. 1999). The released C-terminal segment of EIN2 is translocated to the nucleus and activates the transcription of ethylene-responsive genes (Qiao et al. 2012). Consistent with this model, ethylene receptors bearing a mutation in the amino acid sequences required for ethylene binding (e.g., etr1-1 and Cm-ERS1/H70A) are persistently active in the presence of ethylene and

Abbreviations: ACC, 1-amino-cyclopropane-carboxylic acid; AVG, L- $\alpha$-(2-aminoethoxyvinyl)-glycine; EIN, ETHYLENE INSENSITIVE; ETR, ETHYLENE RESPONSE; ERS, ETHYLENE RESPONSE SENSOR; RT-PCR, reverse transcription PCR; GAF, cGMP-specfic phosphodiesterase, adenylyl cyclases and FhlA.

This article can be found at http://www.jspcmb.jp/

Published online March 16, 2016 
confer a dominant ethylene-insensitive phenotype similar to the recessive ein 2 mutant.

Genetic signaling components for ethylene responses have also been characterized in legume plants. In $M$. truncatula, an ethylene insensitive mutant named sickle showed a strong insensitivity to ethylene or ACC, and formed a clustered and dramatically-increased number of nodules (Penmetsa and Cook 1997). The SICKLE gene encodes an orthologous protein to EIN2 in Arabidopsis (Penmetsa et al. 2008). Similarly, a simultaneous suppression of two Lotus EIN2 homologs, LjEIN2-1 and LjEIN2-2, confers a strong ethylene insensitivity and results in a hypernodulation phenotype comparable to the Medicago sickle mutant (Miyata et al. 2013). These results support the importance of ethylene in the negative regulation of nodulation, and indicate the role of EIN2 in the legume ethylene signaling pathway. On the other hand, the soybean ethylene receptor mutant, etr1-1, shows no significant difference in nodulation phenotype when compared with wild-type plants, whereas the etr1-1 mutation is highly tolerant of ACC or ethylene (Schmidt et al. 1999). Nukui et al. conferred ethylene-insensitivity to L. japonicus by overexpressing the mutated melon ethylene receptor $\mathrm{Cm}$-ERS1/H70A (Nukui et al. 2004). The transgenic plants showed a slightly increased nodulation phenotype. Similarly, Lohar et al. transformed Lotus plants with a mutated Arabidopsis ethylene receptor, etr1-1, under the control of CaMV-35S promoter and the transgenic plants were named LjETR1-1 (Lohar et al. 2009). The roots of these transgenic LjETR1-1 plants also showed insensitivity to ethylene and formed an increased number of nodules when compared to those of wild-type plants under ethylene accumulating condition. However, the degree of increased nodulation in transgenic plants bearing mutated melon or Arabidposis ethylene receptors is moderate and not comparable to the defects of Lotus EIN2 genes (Lohar et al. 2009; Miyata et al. 2013; Nukui et al. 2004). Results regarding ethylene receptors were thus not fully consistent with those regarding EIN2.

Because symbiotic nodulation is restricted in legumes, it is possible that ethylene receptors and/ or downstream signaling pathways have specifically evolved for controlling the newly-acquired mechanism in these plants. However, the function of intrinsic ethylene receptors in model legumes (L. japonicus or $M$. truncatula) has not yet been characterized, although the effects of heterologous mutated ethylene receptors were reported for L. japonicus (Lohar et al. 2009; Nukui et al. 2004). In the present study, we screened for Lotus ethylene-insensitive mutants and isolated a novel mutant lacking ethylene responses. As the mutant bears a mutation in the Lotus ETR1 homolog, our results indicate that the intrinsic ethylene receptor engages in both symbiotic and non-symbiotic ethylene signaling pathways.

\section{Materials and methods}

\section{Plant materials and growth conditions}

Mutagenized M2 seeds of L. japonicus ecotype MG-20 for mutant screening were kindly provided from Prof. Norio Suganuma (Hakoyama et al. 2012). These M2 seeds were germinated and grown on half-strength Gamborg's B5 medium (Wako) including $10 \mu \mathrm{M} \mathrm{ACC}$ and grown in an artificially-lit growth cabinet at $24^{\circ} \mathrm{C}$ for $16 \mathrm{~h}$ (light) and $22^{\circ} \mathrm{C}$ for $8 \mathrm{~h}$ (dark). The method for nodulation analysis has been previously described (Nakagawa et al. 2011).

\section{Map-based cloning}

The causal gene of the Ljetr1 mutant was identified by mapbased cloning as described previously (Yano et al. 2009). A total of 87 F2 plants generated by crossing with L. japonicus B-129 Gifu were assessed in a co-segregation analysis with Lotus DNA markers provided by the Lotus Genome Sequencing Project (Kazusa DNA Research Institute). Genomic DNA of LjETR1 was amplified by the primers LjETR1-F (5' ACC GTC TTC ACC AGC TAG CTA-3') and LjETR1-R (5' AAA CAT AAA TAA TCT TTT ATT CAC AAA AAT GG-3') in both the etil and eti2 mutant lines, then sequenced and compared to wild-type LjETR1.

\section{Expression analysis}

Experimental methods for total RNA extraction and real-time PT-PCR analysis were described previously (Nakagawa et al. 2011). The gene specific primers used for LjETR1 were 5' -TTG CTC TTA CGG GAA ACA CC-3' and 5'-CAC CCC TCA TTT TGT CAA CAG-3'.

\section{Results}

It is well known that dark-grown etiolated seedlings show shorter roots and hypocotyls in the presence of ethylene or ACC (Figure 1A). To obtain Lotus ethylene-insensitive mutants, we screened 1,500 EMS (Ethyl methanesulfonate)-mutagenized seeds (ecotype Miyakojima (MG-20)) by focusing on growth tolerance in the presence of $10 \mu \mathrm{M}$ ACC. Two ethylene-insensitive mutants were found, tentatively named eti1 and eti2. The eti1 mutant was backcrossed to its non-parental ecotype Gifu (B-129) for mapping analysis. Interestingly, F1 progenies also showed weak tolerance to ACC similar to the Arabidopsis etr1-1 mutant (Figure 1A) (Chang et al. 1993). In addition, mapping analysis using F2 progenies suggests that the mutation affecting ethylene insensitivity was mapped to a location near TM0436 (10.5 cM) on chromosome 3 (Figure 1C), where the Lotus ETR1 homolog (chr3.CM0634.490.nc) was placed by the Lotus Genome Sequencing Project. We cloned and sequenced the corresponding genomic region of 
A

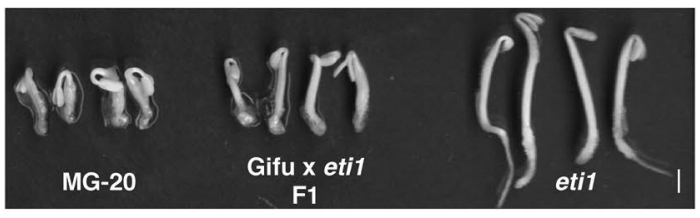

B

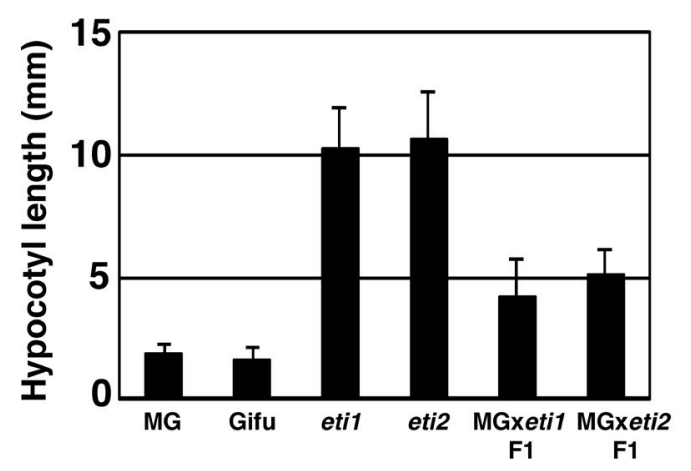

C

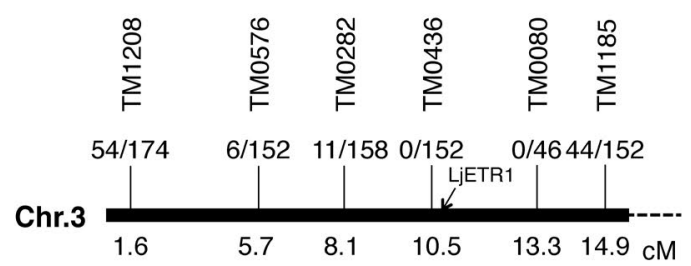

D

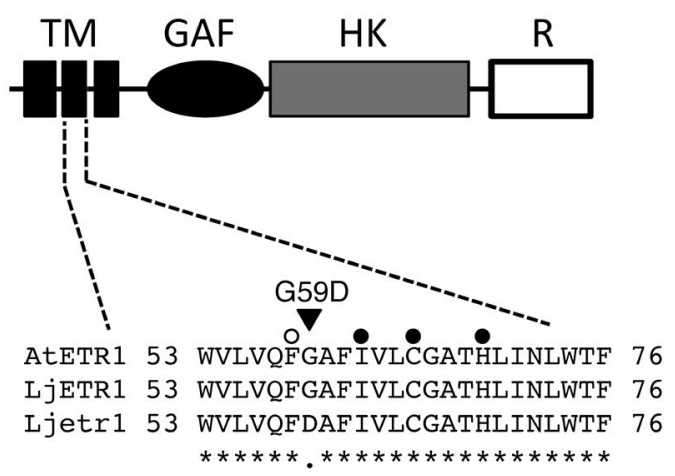

Figure 1. Ethylene-insensitive etil and eti2 mutants show tolerant growth under high concentrations of ACC and bear a mutation in LjETR1. (A) Dark-grown seedlings (5-day-old) of wild-type MG-20, F1 progenies of Gifu and eti1, and eti1 homozygous F2 plants. Bar: $1 \mathrm{~mm}$. (B) Hypocotyl length under $10 \mu \mathrm{M}$ ACC. The data are expressed as means and SDs of more than 10 plants. (C) Genetic map of the LjETR1 region with markers and numbers of recombinant events above the line. The genetic distances of each marker from the terminal marker TM0793 of chromosome 3 are also shown. (D) Protein structure of LjETR1. TM: ethylene binding transmembrane domain containing three hydrophobic segments, GAF: GAF domain, HK: histidine kinase domain, R: receiver domain. The alignment of amino acid sequences in the second hydrophobic segment of ethylene binding domain is also shown. Open and closed circles represent essential residues for ethylene signaling or ethylene binding in AtETR1, respectively.
ETR1 in both eti1 and eti2 mutants and found the same $\mathrm{G}$ to A mutation in both mutants, which conferred an amino acid change from Gly to Asp at protein position 59 in the second hydrophobic segment of the ethylene binding segment (Figure 1D). The region around G59 in ETR1 is well conserved between Lotus and Arabidopsis (Figure 1D). Although the exact role of G59 has not yet been determined, previous studies of Arabidopsis ETR1 predicted the position of this amino acid residue to be in the vicinity of residues essential for both ethylene binding (I62) and ethylene signaling (F58) in the 3D conformation of ETR1 (Wang et al. 2006). We also backcrossed these eti1 and eti2 mutants to parental MG-20 and compared the ethylene insensitivity in their progenies. Both F1 and F2 progenies of etil showed similar phenotypes to those of eti2 (Figure 1B). As these lines may therefore be the siblings from the same M1 plant, we renamed these mutants as Ljetr1. The following analyses used the twice-backcrossed progenies of the eti1 line as Ljetr1.

To evaluate the intensity of the ethylene-insensitive phenotype, homozygous Ljetr1 and the F1 progenies of Ljetr1 and MG-20 were subjected to different
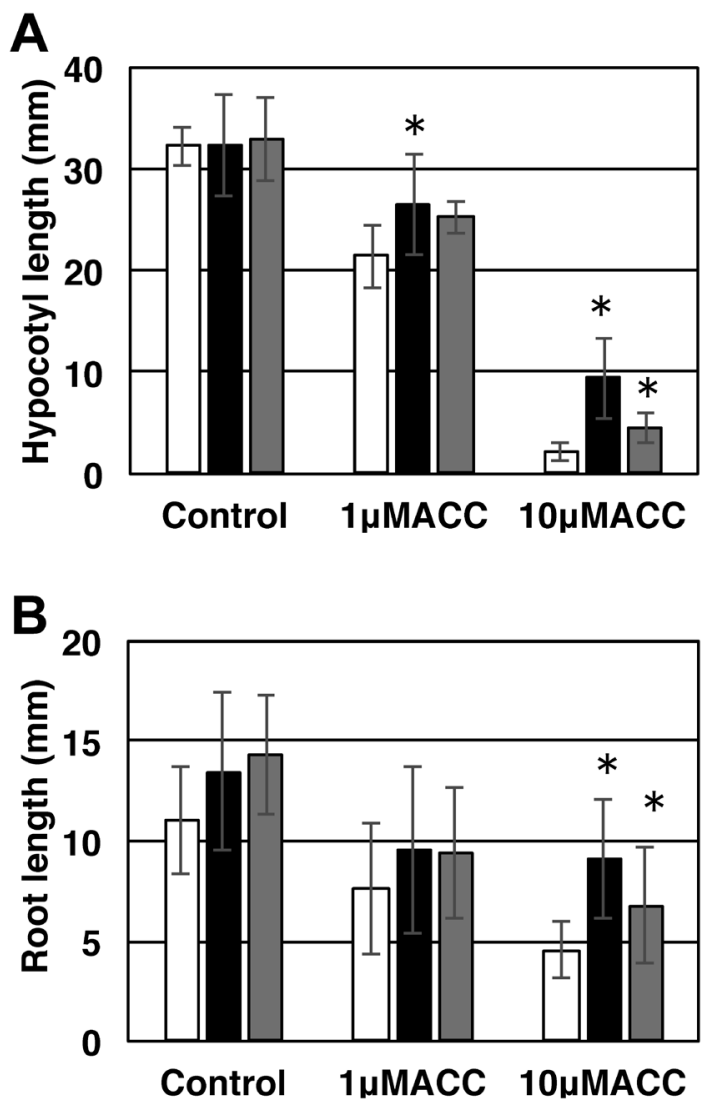

Figure 2. The phenotype of Ljetr1 is more prominent in higher concentrations of ACC. Hypocotyl (A) or root (B) length of MG-20 (open bar), homozygous Ljetr1 (black bar) and F1 progenies of MG-20 and Ljetr1 (gray bar). The data are expressed as means and SDs of more than 7 plants. Black stars indicate significant differences from MG-20 in each condition by Student's $t$-test $(p<0.01)$. 
concentrations of ACC (Figure 2). In the absence of ACC, neither root growth nor shoot growth were significantly different from the parental wild-type plants. Lower concentrations of ACC $(1 \mu \mathrm{M})$ decreased shoot and root growth in both wild-type plants and Ljetr1 mutant. Tolerant growth of Ljetr 1 mutant or F1 progenies was prominent under high concentrations of ACC $(10 \mu \mathrm{M})$. These results indicate that the Ljetr 1 mutant has a decreased response but does not completely lose ethylene responsiveness.

It is well known that ethylene is involved in floral petal abscission. In L. japonicus, pistils and stamens

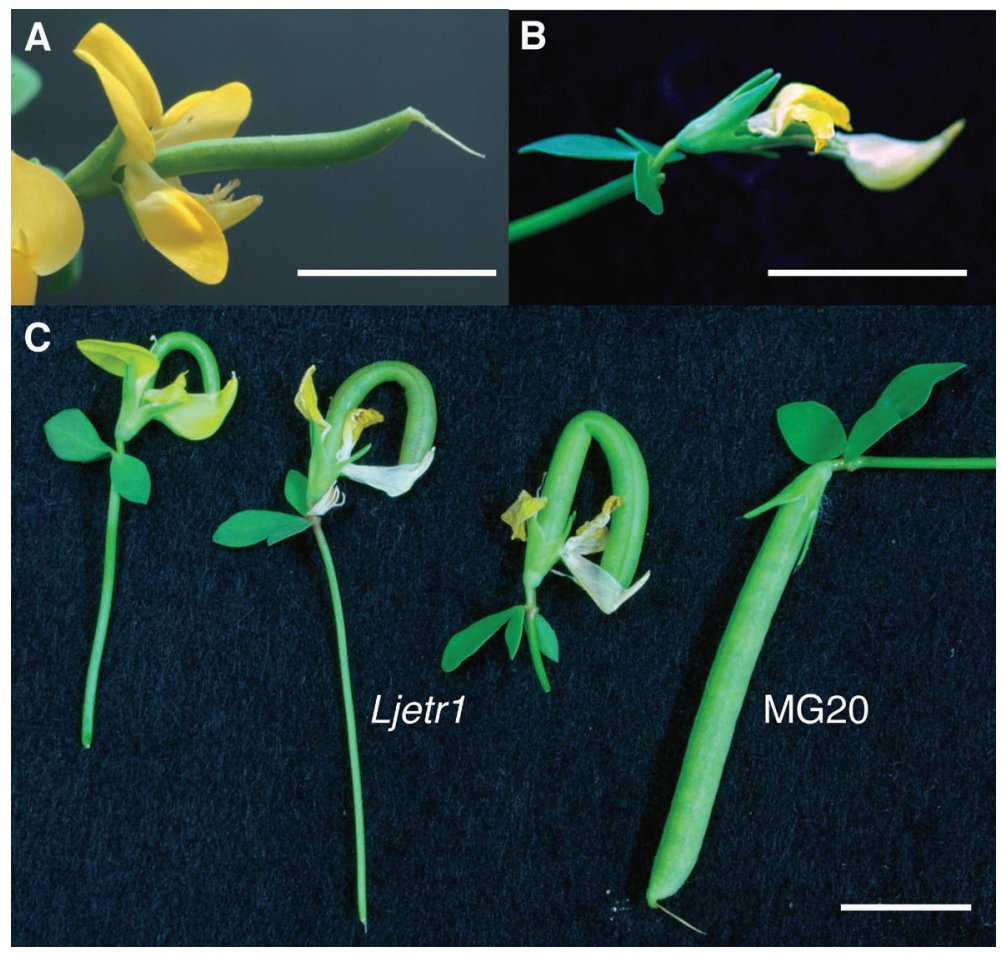

Figure 3. Flowering phenotype of Ljetr1. Petals were withered after the pollination in MG-20 (B) but still fresh in Ljetr1 (A). Pod elongation of Ljetr1 was often interfered with by the persistently-attached keel petal (C). Bars: $1 \mathrm{~cm}$.
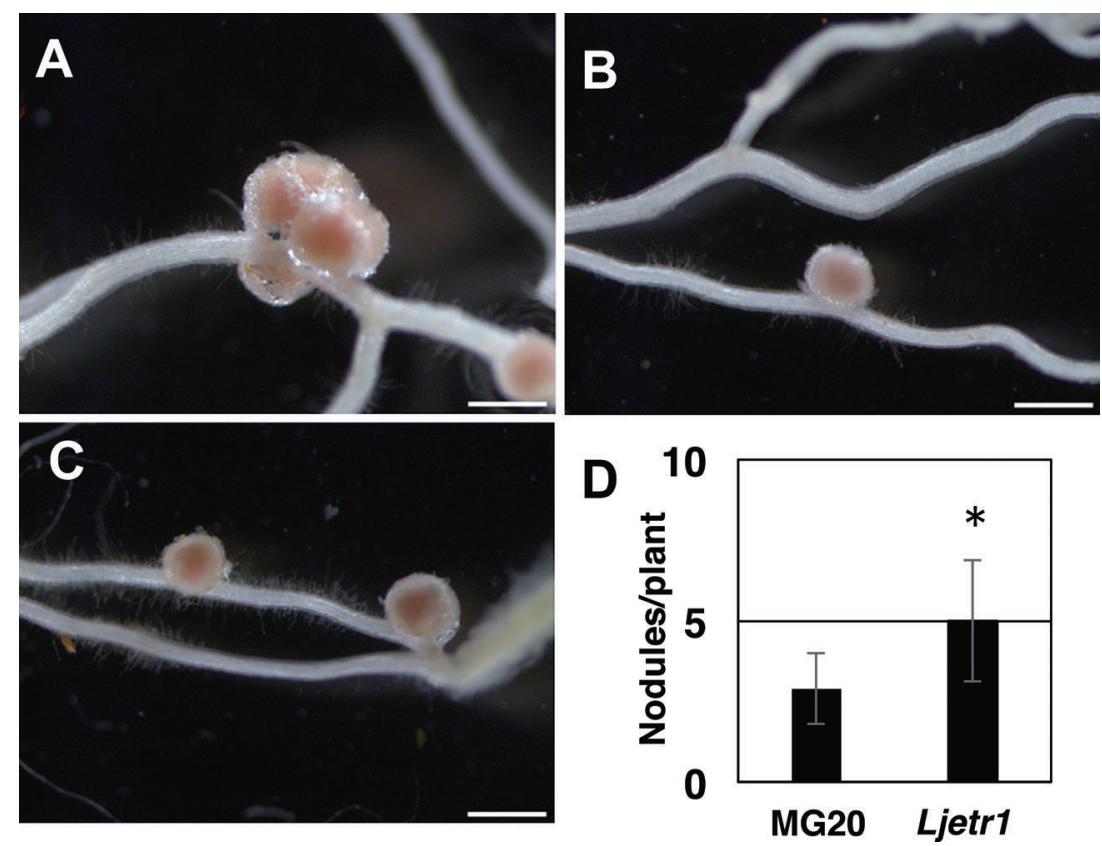

Figure 4. Nodulation phenotype of Ljetr1. Nodules formed on Ljetr1 were often clustered (A) whereas others formed independently (B), similar to wild-type plants (C). D, Number of nodules in MG-20 and Ljetr1 at 23 days after inoculation. The data are expressed as means and SDs of more than 10 plants. The numbers of nodules were statistically different between MG-20 and Ljetr1 mutant $(p<0.01)$. 


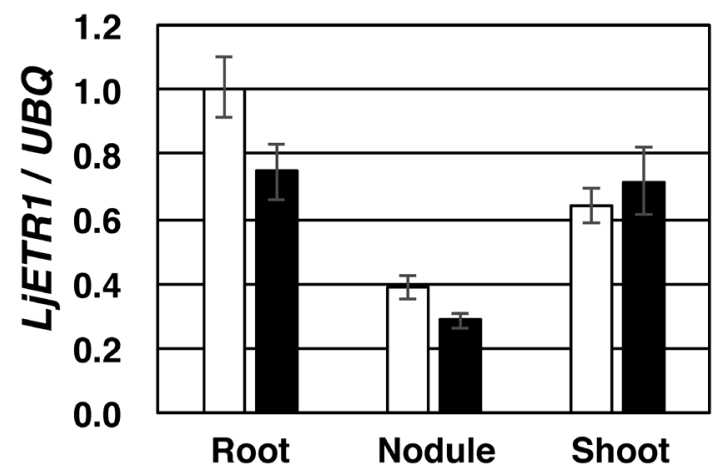

Figure 5. Real time RT-PCR analysis of LjETR1 in both wildtype MG-20 (open bar) and Ljetr1 mutant (black bar). The data are expressed as means and SDs of three biological replications.

are covered by keel petals. After fertilization, the petals wither and detach from flowers before pod elongation (Figure 3B). In contrast, the petals of Ljetr1 remained fresh and stayed in place during the pod elongation process (Figure $3 \mathrm{~A}$ ). As a result, the pod elongation of Ljetr1 was often interfered with, and the pods were bent (Figure 3C).

To investigate the symbiotic phenotype of Ljetr1, the mutant was inoculated with $M$. loti MAFF303099 and its nodulation phenotype was compared with wild-type plants (Figure 4). Ljetr1 mutant often formed clustered nodules (Figure $4 \mathrm{~A}$ ), and the number of nodules was slightly but significantly increased (Figure 4D). However, the degree of increased nodulation was not comparable to EIN2-suppressed plants (Miyata et al. 2013).

The expression pattern of LjETR 1 was investigated by semi-quantitative reverse transcription RT-PCR (Figure 5). LjETR1 was expressed in both shoot and root, and also in nodules. A similar expression level of LjETR1 was also observed in Ljetr1 mutant. These results support the theory that mutated LjETR1 proteins interfere with the intrinsic ethylene signaling pathway and result in the ethylene-insensitive phenotype in Ljetr1 mutant.

\section{Discussion}

In the model legumes L. japonicus and M. truncatula, the function of intrinsic ethylene receptors has not been functionally characterized, although the effects of mutated heterologous ethylene receptors have been investigated. Here, we report that the ethyleneinsensitive Ljetr1 mutant bears a mutation in LjETR1. In Arabidopsis, mutations in I62, C65 or H69 of the second hydrophobic segment of the ethylene-binding domain completely abolish ethylene-binding activity. F58 is not required for ethylene binding, but is essential for ethylene signaling (Wang et al. 2006) (Figure 1D). Therefore, the G59D mutation in Ljetr1 might disturb the conformation of the second hydrophobic segment of the ethylenebinding domain and result in a constitutively active
LjETR1 in the presence of ethylene. On the other hand, the symbiotic phenotype of Ljetr1 (Figure 4) is seemingly weaker than in previously reported LjEIN2-suppressed plants (Miyata et al. 2013). These results might imply the possibility that the G59D mutation is not sufficient to eliminate the ethylene-binding activity or to suppress the ethylene responses. However, note that overexpression of Arabidopsis etr1-1 or mutated melon CmERS1 also showed a phenotype with modestly increased nodulation (Lohar et al. 2009; Nukui et al. 2004). It is possible that ethylene receptor(s) other than LjETR1 also participate in the symbiotic ethylene-signaling pathway.

The phenotype of the Ljetr1 mutant was more prominent at higher concentrations of ethylene (Figure 2). Ethylene receptors are constantly active to suppress the downstream signaling pathways in the absence of ethylene, and most might still be active under low ethylene concentration. Therefore, Ljetr1 mutant might only rarely show a significant difference when compared to wild-type plants in low ethylene conditions (Figure 2). On the contrary, higher concentrations of ethylene inactivated most ethylene receptors and made the effects of the persistently active Ljetr1 protein more pronounced.

Ethylene is engaged in the regulation of plant growth, development, and responses to both biotic and abiotic stresses. Multiple copies of ethylene receptors might reflect such divergent roles of ethylene (Gallie 2015a). Detailed analyses of ethylene receptors in Arabidopsis indicate that some receptors also have specialized physiological functions in addition to a common and overlapping ethylene-signaling suppression activity (Liu et al. 2010; Liu and Wen 2012; Plett et al. 2009; Wilson et al. 2014). In addition, phylogenetic and structural analysis indicated that the composition and structure of ethylene receptors varies among land plants (Gallie 2015b). Land plants may thus have been coordinating ethylene-signaling pathways to adapt to environmental changes through the modification of ethylene receptors (Gallie 2015a). Legumes may also have adapted their ethylene-signaling pathways to control the newly acquired mechanism of symbiotic nodulation. Interestingly, the amino acid sequences of the $\mathrm{N}$-terminal ethylene binding domain and GAF (cGMP-specfic phosphodiesterase, adenylyl cyclases and FhlA) domain are well conserved between LjETR1 and AtETR1 (identity: $90 \%$, similarity $98 \%$ ), whereas the similarity in the histidine kinase and receiver domain (by which ethylene receptors control the downstream signaling pathway) between these genes is rather low (identity: $71 \%$, similarity $94 \%)$. It is intriguing to hypothesize that the modification of the histidine kinase and/or receiver domain enables LjETR1 to regulate the downstream symbiotic ethylene-signaling pathway more efficiently, although overexpression of the mutated Arabidopsis etr11 or melon Cm-ERS1/H70A can also affect it. Future 
functional analyses of other Lotus ethylene receptors and downstream symbiotic ethylene-signaling pathways can be expected to shed more light on this interpretation.

\section{Acknowledgements}

We are grateful to Norio Suganuma (Aichi University of Education) for providing EMS-mutagenized seeds. We also thank Prof. Shibuya Naoto (Meiji University), Prof. Hanae Kaku (Meiji University) and Prof. Masayoshi Kawaguchi for helpful discussions. We thank Yoshinobu Zin for technical support. This study was supported by a KAKENHI Grant-in-Aid for Scientific Research (C) (No.15K07114) to T.N.

\section{References}

Alonso JM, Hirayama T, Roman G, Nourizadeh S, Ecker JR (1999) EIN2, a bifunctional transducer of ethylene and stress responses in Arabidopsis. Science 284: 2148-2152

Chang C, Kwok SF, Bleecker AB, Meyerowitz EM (1993) Arabidopsis ethylene-response gene ETR1: Similarity of product to two-component regulators. Science 262: 539-544

Gallie DR (2015a) Ethylene receptors in plants-why so much complexity? F1000Prime Rep 7: 39

Gallie DR (2015b) Appearance and elaboration of the ethylene receptor family during land plant evolution. Plant Mol Biol 87: 521-539

Guo H, Ecker JR (2004) The ethylene signaling pathway: new insights. Curr Opin Plant Biol 7: 40-49

Hakoyama T, Oi R, Hazuma K, Suga E, Adachi Y, Kobayashi M, Akai R, Sato S, Fukai E, Tabata S, et al. (2012) The SNARE protein SYP71 expressed in vascular tissues is involved in symbiotic nitrogen fixation in Lotus japonicus nodules. Plant Physiol 160: 897-905

Liu Q, Wen CK (2012) Arabidopsis ETR1 and ERS1 differentially repress the ethylene response in combination with other ethylene receptor genes. Plant Physiol 158: 1193-1207

Liu Q, Xu C, Wen CK (2010) Genetic and transformation studies reveal negative regulation of ERS1 ethylene receptor signaling in Arabidopsis. BMC Plant Biol 10: 60

Lohar D, Stiller J, Kam J, Stacey G, Gresshoff PM (2009) Ethylene insensitivity conferred by a mutated Arabidopsis ethylene receptor gene alters nodulation in transgenic Lotus japonicus. Ann Bot (Lond) 104: 277-285

Miyata K, Kawaguchi M, Nakagawa T (2013) Two distinct EIN2 genes cooperatively regulate ethylene signaling in Lotus japonicus. Plant Cell Physiol 54: 1469-1477

Nakagawa T, Kaku H, Shimoda Y, Sugiyama A, Shimamura M, Takanashi K, Yazaki K, Aoki T, Shibuya N, Kouchi H (2011)
From defense to symbiosis: Limited alterations in the kinase domain of LysM receptor-like kinases are crucial for evolution of legume-Rhizobium symbiosis. Plant J 65: 169-180

Nukui N, Ezura H, Minamisawa K (2004) Transgenic Lotus japonicus with an ethylene receptor gene Cm-ERS1/H70A enhances formation of infection threads and nodule primordia. Plant Cell Physiol 45: 427-435

Nukui N, Ezura H, Yuhashi K, Yasuta T, Minamisawa K (2000) Effects of ethylene precursor and inhibitors for ethylene biosynthesis and perception on nodulation in Lotus japonicus and Macroptilium atropurpureum. Plant Cell Physiol 41: 893-897

Oldroyd GE, Engstrom EM, Long SR (2001) Ethylene inhibits the Nod factor signal transduction pathway of Medicago truncatula. Plant Cell 13: 1835-1849

Penmetsa RV, Cook DR (1997) A legume ethylene-insensitive mutant hyperinfected by its rhizobial symbiont. Science 275 : 527-530

Penmetsa RV, Uribe P, Anderson J, Lichtenzveig J, Gish JC, Nam YW, Engstrom E, Xu K, Sckisel G, Pereira M, et al. (2008) The Medicago truncatula ortholog of Arabidopsis EIN2, sickle, is a negative regulator of symbiotic and pathogenic microbial associations. Plant J 55: 580-595

Plett JM, Mathur J, Regan S (2009) Ethylene receptor ETR2 controls trichome branching by regulating microtubule assembly in Arabidopsis thaliana. J Exp Bot 60: 3923-3933

Qiao H, Shen Z, Huang SS, Schmitz RJ, Urich MA, Briggs SP, Ecker JR (2012) Processing and subcellular trafficking of ER-tethered EIN2 control response to ethylene gas. Science 338: 390-393

Schmidt JS, Harper JE, Hoffman TK, Bent AF (1999) Regulation of soybean nodulation independent of ethylene signaling. Plant Physiol 119: 951-960

Suzaki T, Yoro E, Kawaguchi M (2015) Leguminous plants: Inventors of root nodules to accommodate symbiotic bacteria. Int Rev Cell Mol Biol 316: 111-158

Wang W, Esch JJ, Shiu SH, Agula H, Binder BM, Chang C, Patterson SE, Bleecker AB (2006) Identification of important regions for ethylene binding and signaling in the transmembrane domain of the ETR1 ethylene receptor of Arabidopsis. Plant Cell 18: $3429-3442$

Wilson RL, Kim H, Bakshi A, Binder BM (2014) The Ethylene Receptors ETHYLENE RESPONSE1 and ETHYLENE RESPONSE2 Have Contrasting Roles in Seed Germination of Arabidopsis during Salt Stress. Plant Physiol 165: 1353-1366

Yano K, Shibata S, Chen WL, Sato S, Kaneko T, Jurkiewicz A, Sandal N, Banba M, Imaizumi-Anraku H, Kojima T, et al. (2009) CERBERUS, a novel U-box protein containing WD-40 repeats, is required for formation of the infection thread and nodule development in the legume-Rhizobium symbiosis. Plant $J 60$ : 168-180 